\title{
FOMENTO DEL TRABAJO FUERA DEL AULA CON EVALUACIÓN ENTRE PARES Y TUTORÍAS INTEGRALES DE GRUPO
}

\section{HOMEWORK ENCOURAGEMENT BY MEANS OF PEER REVIEWED ACTIVITIES AND GLOBAL GROUP TUTORIALS}

\author{
Francisco J. Castillo-Ruiz*, Sergio Castro-García, \\ Juan Agüera-Vega \\ *g62caruf@uco.es
}

Received: 30/06/2017 Accepted: 25/06/2018

\begin{abstract}
Resumen
Se parte de la detección de una serie de carencias en la adquisición de conocimientos en la asignatura Motores y Máquinas de $2 .^{\circ}$ curso del Grado en Ingeniería Agroalimentaria y del Medio Rural. Para dirigir y motivar al alumnado de cara a la superación de la asignatura se organizaron dos prácticas fuera del aula (P2 y P3), de la misma naturaleza que los tres tipos de ejercicios de examen (P1, P2 y P3). La corrección de las prácticas se hizo mediante evaluación por pares y posterior revisión del profesorado. La realización de las dos prácticas tuvo un seguimiento desigual, con una tasa de evaluación de 33 y $22 \%$ respectivamente, mientras que la tasa de éxito fue muy elevada, 87 y $97 \%$. En cuanto a los resultados del examen, la tasa de evaluación fue del 59 y $55 \%$, y la tasa de éxito del 50 y $36 \%$, inferior a la registrada en las prácticas. Sin embargo la tasa de rendimiento fue similar entre el examen $(30$ - $20 \%$ y las prácticas (29 y $12 \%)$. Los resultados de los exámenes del curso $2016 / 17$ fueron mejores que en los exámenes del curso 2015/16, por lo tanto se ha cumplido el objetivo de mejorar el porcentaje de alumnos que superan la asignatura, mejorando o manteniendo el rendimiento. Por último, la tasa de evaluación no mostró un resultado satisfactorio en las prácticas, por lo que se hace necesario incidir entre el alumnado en la importancia de estas actividades.
\end{abstract}

Palabras clave: Pedagogía del error, aprendizaje fuera del aula, trabajo fuera del aula, tasa de éxito, tasa de rendimiento.

\begin{abstract}
Firstly, it has been detected some deficiencies in the acquisition of knowledge for the subject "Motores y Máquinas" corresponding to the $2^{\text {nd }}$ year of the Degree in Agri-food Engineering and Rural Environment. Two homework practices (P2 and P3), similar to the three types of exam exercises (P1, P2 and P3) were organized to tutorize and motivate students in order to pass the subject. Practices correction was done through peer evaluation and subsequent revision of the teaching staff. The performance of the two practices was unevenly followed, with an evaluation rate of 33 and $22 \%$ respectively, while the success rate was very high, 87 and $97 \%$. Regarding the results of the examination, the evaluation rate was 59 and $55 \%$ and the success rate was of 50 and 36\% for P2 and P3 respectively, lower than that recorded in the practices. However, the rate of performance was similar between exam $(30-20 \%)$ and practices (29 and 12\%). The results of the 2016/17 exams were better than in the examinations of the 2015/16 course, therefore the objective of improving the percentage of students who pass the subject, improving or maintaining the performance has been accomplish. Finally, the evaluation rate did not show a satisfactory result for the practices, for this reason, it is necessary to persuade the students to the importance of these activities.
\end{abstract}

Keywords: Pedagogy of error correction, out of classroom learning, homework, success rate, performance rate.

\section{INTRODUCCIÓN}

El avance en la aplicación del conocimiento, y las técnicas pedagógicas en las asignaturas correspondientes a los Grados de Ingeniería Agroalimentaria y del Medio Rural, Enología e Ingeniería Forestal, es una necesidad impuesta por el mercado laboral. En este sentido, la Universidad de Córdoba se propone avanzar en la mejora de la calidad de la enseñanza para proveer al mercado laboral de profesionales de alta cualificación que se ajusten a la demanda del mismo. Además, el avance de la internacionalización de la Universidad de Córdoba debe realizarse mediante la atracción de alumnos del Espacio Europeo de Educación Superior (E.E.E.S) y de terceros países. El proceso de atracción de alumnos internacionales debe tener como base la calidad de la enseñanza y la adecuación de las titulaciones a las necesidades reales de su desempeño profesional.

El proceso de aprendizaje requiere de una base previa de conocimientos en materias transversales que en ocasiones no ha sido correctamente adquirida por los alumnos. En este sentido se ha observado una carencia en este campo en los alumnos de las titulaciones de Grado de Ingeniería Agroalimentaria y del Medio Rural, Enología y Grado de Ingeniería Forestal. Además, se ha detectado una falta de aprovechamiento de las horas de trabajo fuera del aula por parte del alumnado, debido fundamentalmente al predominio de la lección magistral como método de transmisión de conocimientos (CIDUA, 2005). Este trabajo fuera del aula, debe proporcionar al alumno la oportunidad de explorar la materia con mayor profundidad, adquiriendo por un lado el nivel adecuado en las competencias requeridas para superar la asignatura, y por otro lado conocimientos prácticos aplicables a situaciones reales, que permitan al alumno estar preparado de cara a una posible 
inmersión profesional en una posible relación universidad-empresa durante la realización de su futuro trabajo fin de grado (Requena et al., 2018).

Para solucionar estos problemas, el Área de conocimiento de Ingeniería Agroforestal está comprometida con la mejora de la calidad educativa basada en las metodologías activas centradas en el alumnado a través de actividades académicamente dirigidas. Estas actividades tienen como objetivo principal la aplicación del conocimiento teórico a la práctica mediante la realización de ejercicios y actividades prácticas. Además, se contribuye mantener un Grupo Docente activo e innovador en las asignaturas del área, capaz de difundir los resultados de las nuevas experiencias educativas llevadas a cabo.

Aumentar el autoaprendizaje entre el alumnado mediante la búsqueda, selección e interpretación de información en los buscadores y bases de datos específicas es un aspecto fundamental para la formación del alumnado. La adquisición de la competencia de "acceso y uso de la información" es de gran importancia en la preparación de los futuros profesionales, y dota al alumnado de la capacidad para enfrentarse a una gran diversidad de problemas en el ámbito profesional. Por último, el plurilingüismo es identificado como una competencia esencial para el desempeño de la actividad de un titulado superior. En este sentido, el fomento del conocimiento de otras lenguas se puede potenciar mediante la búsqueda de información en fuentes y bases de datos en otro idioma diferente de la lengua materna del alumnado.

El fomento del trabajo fuera del aula en las asignaturas de segundo curso de las citadas titulaciones permite al alumno adquirir los conocimientos y destrezas necesarios para superar la asignatura y para desarrollar su carrera profesional con un nivel adecuado en el campo de la mecanización agraria. El desarrollo de tareas fuera del aula es uno de los pilares del E.E.E.S. y por tanto una obligación del alumno. Sin embargo, la escasa implantación del autoaprendizaje entre el alumnado durante los primeros cursos de grado hace que, en muchos casos, no se aproveche este tiempo de trabajo, y en numerosas ocasiones este hecho conlleva la no superación de la asignatura por parte del alumno. Además, es conveniente aprovechar la pedagogía del error como método de aprendizaje (Lomas, 2016) en el que el alumno pierde el miedo al fracaso, sustituyéndolo por la visión de una oportunidad para aprender.

Un paso más en el aprendizaje del alumnado, radica en la evaluación entre iguales, que permite al alumno aprender no sólo de sus propios errores, sino también de los errores de sus compañeros. En este sentido la evaluación entre pares siempre debe estar supervisada por el profesor, para evitar que la inexperiencia de los alumnos de lugar a una evaluación injusta hacia sus compañeros, y también puede hacerse mediante elaboración y aplicación de rúbricas a nivel grupal (Calvo Sastre, 2018). Además, la evaluación por pares genera un cambio en las relaciones de poder tutor-alumno, reduciendo los tiempos de respuesta respecto a la corrección de todos los ejercicios por parte del docente, y aportando comentarios más clarificadores y más cercanos a los alumnos (Vera-Cazorla, 2014). Además este tipo de evaluación puede aplicarse a trabajos colaborativos (Santana-Quintana y Vera-Cazorla, 2013).

\section{OBJETIVOS}

La evaluación realizada de los resultados obtenidos en convocatorias y proyectos de innovación docentes anteriores han puesto de manifiesto la importancia de la participación y motivación del alumno para el aprovechamiento y éxito en las asignaturas de segundo curso. El presente artículo de innovación docente propone avanzar en la aplicación de este conocimiento adquirido, con una mayor actividad, implicación y autonomía del alumnado en el desarrollo de los contenidos prácticos de la asignatura.

El presente estudio tiene como objetivo principal evaluar el aumento del porcentaje de alumnos que superan la asignatura y el afianzamiento de las competencias adquiridas, con la introducción prácticas fuera del aula y su metodología de corrección. Además, se ha buscado una mejora de la implicación del alumnado en la asignatura y dotar a la misma de una aplicación práctica del conocimiento teórico.

Dado que en Cirugía gran parte del contenido temático consiste en el aprendizaje de técnicas (anudados, tipos de intervenciones, posiciones del paciente, etc), el uso de las nuevas tecnologías como el aprendizaje multimedia resulta esencial.

\section{Metodología}

Actualmente, en la asignatura "Motores y máquinas" de segundo curso del Grado en Ingeniería Agroalimentaria y del Medio Rural se realizan una serie de prácticas en las que se explican tres tipos de problemas que luego se evalúan en el examen. Estas prácticas se imparten fomentando la participación del alumnado, incluyendo en todo caso un problema que debe ser resuelto por el alumno al final de la clase. Sin embargo, es frecuente que durante cada práctica los alumnos no adquieran los conocimientos requeridos, Por lo tanto, en el presente trabajo se plantea reforzar estas prácticas con tareas realizadas fuera del aula por los alumnos.

De los tres tipos de problema de examen (P1, P2 y P3), se han diseñado prácticas para realizar como trabajo fuera del aula en dos de ellos (P2 y P3). Para ello, se ha elaborado un enunciado de problema simulando un examen y se ha puesto a disposición del alumnado en la plataforma Moodle después de realizar la práctica de aula correspondiente. Se ha dado dos semanas de plazo para la ejecución del problema por parte del alumnado como tarea en horario fuera del aula, disponiendo 
el profesorado de la asignatura de un extenso horario de tutorías durante ese periodo de tiempo. Una vez realizada la práctica, el alumno la envía a través de la plataforma Moodle para proceder a su corrección.

En primer lugar, se preparó una lista de los problemas entregados asignando un número a cada uno de ellos, y se distribuyó entre los alumnos para que realizasen una corrección por pares, asignando una nota numérica en escala de 0 a 10 . Posteriormente, el profesorado realizó una corrección del problema entregado y de la corrección realizada, dos notas definitivas, una para el alumno que había realizado la práctica, y otra para el alumno que había corregido la práctica. Estas notas junto con las correspondientes sugerencias y correcciones fueron enviadas a través de la plataforma Moodle.

La metodología empleada tuvo una fuerte componente participativa del alumnado, tanto durante la realización de las prácticas fuera del horario lectivo, como en la dedicación del tiempo necesaria fuera del aula. Esta estrategia se planteó debido a la importante carga de trabajo fuera del aula que demandan las titulaciones dentro del E.E.E.S., y que en ocasiones queda infrautilizada por el alumno, principalmente al comienzo del cuatrimestre, generando carencias en los conocimientos adquiridos que en ocasiones llevan al alumno a no superar la asignatura.

Posteriormente se realizó el análisis tanto de las notas obtenidas en la práctica y corrección de la misma, como las notas obtenidas en las distintas convocatorias de examen. Para realizar este análisis se emplearon los indicadores propuestos para analizar los resultados de la asignatura (MINECD, 2014).

Tasa de rendimiento $=\frac{\text { Alumnos que superan la asignatura }}{\text { Alumnos matriculados }} \times 100$

Tasa de éxito $=\frac{\text { Alumnos que superan la asignatura }}{\text { Alumnos presentados }} \rtimes 100$

Tasa de evaluación $=\frac{\text { Alumnos presentados }}{\text { Alumnos matriculados }} \times 100$

Con estos tres indicadores se ha evaluado el efecto de las prácticas diseñadas e implementadas en la asignatura. Finalmente también se ha empleado la nota media de los alumnos participantes como un indicador tanto para las prácticas fuera del aula, como para los exámenes.

Se evaluaron las tasas especificadas y el rendimiento académico como nota media para los exámenes de los cursos 2016/17 y 2015/16, teniendo en cuenta que en el segundo caso no se había puesto en práctica las tareas fuera del aula. Se midieron estos valores para las convocatorias de exámenes de enero (1. a) y febrero (2. ${ }^{a}$ ), así como para la realización de la práctica fuera del aula y corrección de la misma.

\section{RESULTADOS OBTENIDOS}

Las prácticas han registrado un nivel de participación bajo, especialmente en la P3. Sin embargo, la nota media y la tasa de éxito han sido muy elevadas en las prácticas, mientras que, en el examen, estas tasas han sido sensiblemente inferiores (Tabla 1). Entre otros factores, esto puede deberse a que en el trabajo fuera del aula, el alumno dispone de acceso a todo el material de la asignatura, pudiendo además acceder a contenidos on-line, mientras que en el examen sólo dispone de un formulario.

La ejecución de las actividades descritas no ha mostrado una influencia clara sobre la tasa de éxito en los tipos de problemas estudiados para el examen final de la asignatura, sin embargo, si se observaron mejores tasas de evaluación en los exámenes del curso objeto de estudio con respecto a la convocatoria correspondiente del año anterior (Tabla 1). En este sentido, la realización de tareas prácticas con un buen resultado a nivel de evaluación, ha animado a un mayor número de alumnos a enfrentarse a los exámenes de la asignatura. Además, la nota media del curso 2016/17 ha sido superior o igual a la nota media obtenida para el mismo ejercicio en la misma convocatoria para el curso 2015/16. Se requiere un estudio más prolongado en el tiempo para concluir que la realización de tareas fuera del aula ha mejorado la calificación media de los alumnos excepto para el tipo de ejercicio P3 en la primera convocatoria de examen.

Tabla 1. Indicadores de las prácticas realizadas y exámenes del año en curso y el anterior para los tipos de ejercicios considerados.

\begin{tabular}{|c|c|c|c|c|c|c|c|}
\hline Actividad & Indicador & Práctica & Corrección & $\begin{array}{c}\text { Examen } \\
1 .^{a} \text { conv. } \\
16 / 17 \\
\end{array}$ & $\begin{array}{c}\text { Examen } \\
2 .^{a} \text { conv. } \\
16 / 17 \\
\end{array}$ & $\begin{array}{c}\text { Examen } \\
1 .^{a} \text { conv. } \\
15 / 16\end{array}$ & $\begin{array}{c}\text { ixamen } 2 .^{2} \\
\text { conv. } \\
15 / 16\end{array}$ \\
\hline \multirow{2}{*}{ P2 } & $\begin{array}{c}\text { Tasa de } \\
\text { evaluación }(\%)\end{array}$ & 33 & 23 & 58 & 48 & 46 & 29 \\
\hline & $\begin{array}{c}\text { Tasa de éxito } \\
(\%)\end{array}$ & 87 & 96 & 78 & 69 & 77 & 80 \\
\hline
\end{tabular}




\begin{tabular}{|c|c|c|c|c|c|c|c|}
\hline & $\begin{array}{c}\text { Tasa de } \\
\text { rendimiento } \\
(\%)\end{array}$ & 21 & 22 & 45 & 33 & 45 & 31 \\
\hline & Nota media & 7,5 & 8,8 & 6,7 & 5,4 & 5,1 & 4 \\
\hline \multirow{4}{*}{ P3 } & $\begin{array}{c}\text { Tasa de } \\
\text { evaluación }(\%)\end{array}$ & 22 & 12 & 58 & 47 & 46 & 28 \\
\hline & $\begin{array}{c}\text { Tasa de éxito } \\
(\%)\end{array}$ & 97 & 100 & 39 & 54 & 50 & 13 \\
\hline & $\begin{array}{c}\text { Tasa de } \\
\text { rendimiento } \\
(\%)\end{array}$ & 29 & 12 & 22 & 25 & 30 & 5 \\
\hline & Nota media & 9 & 9,3 & 4,1 & 5 & 4,1 & 3,1 \\
\hline
\end{tabular}

\section{UTILIDAD/ANÁLISIS}

La nota obtenida en el examen final puede variar en función de otras variables como son la asistencia a clase, curso y tipo de materia (Pérez y Graell, 2004), así como la interacción entre estas variables. Por otro lado, variables como la motivación, estrategias de aprendizaje o el clima de trabajo y estudio del centro también se han mostrado como factores clave en el rendimiento académico de los alumnos (Garrido-Macias et al., 2013). En este sentido, la realización de prácticas en el aula y la recomendación de trabajos fuera de la misma fomentan un clima de trabajo y aprendizaje que puede influir positivamente en el rendimiento académico de los alumnos. Otras alternativas como la puesta en práctica del aula invertida o "flipped classroom", ha mostrado eficacia a la hora de mejorar significativamente los resultados del alumnado de cara al test de conocimientos (Gahete et al., 2018).

Si se compara el examen atendiendo a los parámetros globales y no por ejercicio, sí que se observa una mejora en todos los campos (Tasa de evaluación, de éxito y de rendimiento) del curso 2016/17 con respecto al curso 2015/16, excepto en la nota media de la segunda convocatoria, que ha resultado equivalente (Tabla 2). En este sentido también se observa un efecto negativo a medida de se avanza en las convocatorias, de tal forma que las distintas tasas y rendimiento académico decae en las convocatorias más avanzadas. Este efecto, puede deberse al nivel de motivación del alumnado. Aquellos alumnos más motivados tienden a presentarse en la primera convocatoria, mientras que los alumnos con un nivel de motivación más bajo suelen presentarse en la segunda o sucesivas convocatorias.

Tabla 2. Indicadores globales para los exámenes en el curso objeto de estudio y el anterior.

\begin{tabular}{ccccc}
\hline Parámetro & $\begin{array}{c}\text { Examen 1. } \\
\text { conv. 16/17 }\end{array}$ & $\begin{array}{c}\text { Examen 2. } \\
\text { conv. 16/17 }\end{array}$ & $\begin{array}{c}\text { Examen 1. } \\
\text { conv. 15/16 }\end{array}$ & $\begin{array}{c}\text { Examen 2. }^{\mathbf{a}} \\
\text { conv. 15/16 }\end{array}$ \\
\hline $\begin{array}{c}\text { Tasa de evaluación } \\
(\%)\end{array}$ & 59 & 55 & 46 & 30 \\
$\begin{array}{c}\text { Tasa de éxito (\%) } \\
\text { Tasa de }\end{array}$ & 50 & 36 & 27 & 20 \\
rendimiento (\%) & 30 & 20 & 16 & 7 \\
Nota media & 4,8 & 3,5 & 3,4 & 3,5 \\
\hline
\end{tabular}

\section{CONCLUSIONES/DISCUSIÓN}


En los exámenes del curso 2016/17, los tres indicadores considerados fueron mejores, y la nota media igual o mejor que en los exámenes del curso 2015/16. Por lo tanto, se ha cumplido el objetivo de mejorar el porcentaje de alumnos que superan la asignatura, mejorando o manteniendo el rendimiento. Por último, la tasa de evaluación en las tareas de corrección de las prácticas fue muy inferior a la registrada en las prácticas, por lo que se concluye que es necesario incidir en la importancia de la pedagogía del error entre el alumnado para que entiendan la realización de estos ejercicios como una oportunidad de aprendizaje y no como una tarea tediosa.

\section{BIBLIOGRAFÍA}

1. Calvo Sastre, A. 2018. La evaluación entre iguales a través del diseño de rúbricas en la formación de educadores y educadoras sociales. I Congreso Virtual Internacional de Innovación Docenta Universitaria We teach \& We learn. Universidad de Córdoba, 20 y 21 de junio de 2018. Córdoba. España.

2. CIDUA. 2005. Informe sobre innovación de la docencia en las universidades andaluzas. (Acceso el 27 de abril de 2017 ). www.uma.es/eees/images/informe innovacion_docencia_univ_andaluzas cidua.doc

3. Gahete, M. D. Burón, M. I. y Luque, R. M. 2018. Experiencia de aula invertida como herramienta de aprendizaje en másteres universitarios en el área de Biología Celular. I Congreso Virtual Internacional de Innovación Docenta Universitaria We teach \& We learn. Universidad de Córdoba, 20 y 21 de junio de 2018. Córdoba. España.

4. Garrido Macias, M. Jiménez Luque, N. Landa Sánchez, A. Páez Espinar, E. y Ruiz Barranco, M. 2013. Factores que influyen en el rendimiento académico: La motivación como papel mediador en las estrategias de aprendizaje y clima escolar. Revista electrónica de investigación Docencia Creativa 2: 17-25.

5. Lomas, C. 2016. Pedagogía del error. Aula de secundaria, 17, pp: 7. (Acceso el 14 de octubre de 2017. http://www.octaedro.com/pdf/Pedagogiadelerror.pdf

6. MINECD. 2014. Anuario de indicadores universitarios. (Acceso el 14 de febrero de 2017). https://www.educacion.gob.es/educabase/tabla.do?path=/Universitaria/Indicadores/2014/2_Academico/1_Grado/10/\&file= EIU02102.px\&type $=$ pcaxis\&L=0\%3C/font $\% 3 \mathrm{E}$

7. Pérez, J. y Graell, S. 2004. Asistencia a clase y rendimiento académico en estudiantes de medicina. La experiencia de la Universidad Autónoma de Barcelona. Educación médica 7: 85-90.

8. Requena, F. Escribano, B. M. Martinez-Marín, A. L. Camacho, R. Castejón, C. y Agüera, E. I. 2018. La importancia de la relación Universidad-Empresa en los trabajos fin de grado. I Congreso Virtual Internacional de Innovación Docenta Universitaria We teach \& We learn. Universidad de Córdoba, 20 y 21 de junio de 2018. Córdoba. España.

9. Santana-Quintana, M. C. y Vera-Cazorla, M. J. 2013. La utilización de rúbricas para la evaluación del trabajo colaborativo del alumnado en las wikis. Revista de Lingüística y Lenguas Aplicadas 8: 120-28.

10. Vera-Cazorla, M. J. 2014. La evaluación formativa por pares en línea como apoyo para la enseñanza de la expresión escrita persuasiva. Revista de educación a distancia 43, 15-Nov-2014. 